\title{
The Energy Momentum Spectrum and Vacuum Expectation Values in Quantum Field Theory, II
}

\author{
JAMES GLimm* \\ Courant Institute of Mathematical Sciences, New York University, New York, N. Y. \\ ARTHUR JAFFE ${ }^{\star \star}$ \\ Lyman Laboratory of Physics, Harvard University, Cambridge, Mass.
}

Received February 22, 1971

\begin{abstract}
We prove that the $\mathscr{P}(\varphi)_{2}$ quantum field theory satisfies the spectral condition. The space time translation $a=(x, t)$ is implemented by the unitary group $U(a)$ $=\exp (i t H-i x P)$, and the joint spectrum of the energy operator $H$ and the momentum operator $P$ is contained in the forward cone. We also obtain bounds on certain vacuum expectation values of products of field operators. Our proofs involve an analysis of the limit $V \rightarrow \infty$ for approximate theories in a periodic box of volume $V$. Assuming the existence of a uniform mass gap, we are able to establish all the Wightman axioms with the exception of the Lorentz invariance of the vacuum.
\end{abstract}

\section{Introduction}

We study a boson quantum field $\varphi$ with a polynomial self interaction $\mathscr{P}(\varphi)$ in two dimensional space time. This theory provides an example of all the Haag-Kastler axioms and many of the Wightman axioms for quantum field theory. In this paper we prove that the energy-momentum spectrum lies in the forward cone. In addition, we prove bounds on the vacuum expectation values of products of the differentiated field operators $\partial_{t} \varphi(x, t)=\varphi_{t}$ and $\partial_{x} \varphi(x, t)=\varphi_{x}$. Three of the Wightman axioms remain open problems for the $\mathscr{P}(\varphi)_{2}$ theory. They are the invariance of the vacuum under Lorentz rotations, the uniqueness of the vacuum and the existence of vacuum expectation values of products of the field $\varphi(x, t)$ (without differentiation). Assuming the existence of a mass gap, we verify the latter two of these three missing axioms in Section 4.

The $\mathscr{P}(\varphi)_{2}$ theory is obtained as a limit of cutoff or approximate field theories. We have previously considered two different space cutoff

* Supported in part by the Air Force Office of Scientific Research, Contract AF $49(638)-1719$.

$\star \star$ Alfred P. Sloan Foundation Fellow. Supported in part by the Air Force Office of Scientific Research, Contract F 44620-70-C-0030.

1 Commun. math. Phys., Vol. 22 
procedures: a "box" cutoff and a "coupling constant" cutoff. In the "coupling constant" cutoff, the coupling constant $\lambda$ is replaced by a space dependent coupling constant $\lambda g(x)$. For instance, $g(x)$ might equal the characteristic function of a bounded interval. In the "box" cutoff, the Hamiltonian is translation invariant, but we replace the configuration space $R^{1}$ by the periodic interval $\left[-\frac{1}{2} V, \frac{1}{2} V\right]$ of length $V$.

In [3] we studied the infinite volume limit of the theory with a coupling constant cutoff. We showed that the limit $g \rightarrow 1$ yields a locally Fock vacuum. We now use the same techniques to show that the limit $V \rightarrow \infty$, of box cutoff theories, yields a locally Fock vacuum. The algebra of observables $\mathfrak{U}$ and the Poincaré automorphisms $\sigma_{\{a, \Lambda\}}$ constructed from the two infinite volume limits, $g \rightarrow 1$ or $V \rightarrow \infty$, are identical. Thus we obtain from the limit $V \rightarrow \infty$ a theory incorporating the results previously established for the limit $g \rightarrow 1$. In addition we have new properties for the limit $V \rightarrow \infty$. The spectral condition $0 \leqq H, 0 \leqq H^{2}-P^{2}$ was previously established in the theory with a box cutoff, $V<\infty$, as were the bounds on $\varphi_{t}$ and $\varphi_{x}$ [4]. In the present paper, we transfer these results to the limit $V \rightarrow \infty$.

We describe the approximate theories in more detail. We follow the notation of our Les Houches lectures [5]. The time zero fields in infinite space $R^{1}$ satisfy the canonical commutation relations

$$
\left[\varphi\left(f_{1}\right), \pi\left(f_{2}\right)\right]=i\left\langle f_{1}, f_{2}\right\rangle .
$$

These field operators act on the Fock space $\mathscr{F}$, which is the symmetric tensor algebra over $\mathscr{L}_{2}\left(R^{1}\right)$. For each bounded open interval $\mathscr{B} \subset R^{1}$, we define the algebra $\mathfrak{A}(\mathscr{B})$ of local observables as the von Neumann algebra generated by the operators

$$
\exp \left(i \varphi\left(f_{1}\right)+i \pi\left(f_{2}\right)\right), \quad f_{i} \in \mathfrak{C}_{0}^{\infty}(\mathscr{B}) .
$$

We also use the $C^{*}$-algebra $\mathfrak{A}_{0}(\mathscr{B})$ generated by these operators; $\mathfrak{A}_{0}(\mathscr{B})$ is weakly dense in $\mathfrak{A}(\mathscr{B})$. Let

$$
\begin{aligned}
\mathfrak{U} & =\text { norm closure }\left(\bigcup_{\mathscr{B}} \mathfrak{A}(\mathscr{B})\right), \\
\mathfrak{A}_{0} & =\text { norm closure }\left(\bigcup_{\mathscr{B}} \mathfrak{A}_{0}(\mathscr{B})\right) .
\end{aligned}
$$

The Hamiltonian $H(g)$ with the coupling constant cutoff $g$ is defined by

$$
H(g)=H_{0}+\int: \mathscr{P}(\varphi(x)): g(x) d x-E_{g} .
$$

Here $\mathscr{P}$ is a positive polynomial, $g$ is a positive function with compact support, and $E_{g}$ is chosen so that inf spectrum $H(g)=0$. 
In the periodic box approximation, the field $\varphi_{V}$ satisfies (1.1), namely $\left[\varphi_{V}\left(f_{1}\right), \pi_{V}\left(f_{2}\right)\right]=i\left\langle f_{1}, f_{2}\right\rangle$, provided $f_{i} \in \mathscr{L}_{2}\left(\left[-\frac{1}{2} V, \frac{1}{2} V\right]\right)$. The periodic Fock space $\mathscr{F}_{V}$ is the symmetric tensor algebra over $\mathscr{L}_{2}([-V / 2, V / 2])$. The Hamiltonian $H_{V}$ for the volume $V$ is

$$
H_{V}=H_{0, V}+\int_{-\frac{1}{2} V}^{\frac{1}{2} V}: \mathscr{P}\left(\varphi_{V}(x)\right): d x-E_{V},
$$

where $E_{V}$ is chosen so that inf spectrum $H_{V}=0$.

There is a natural identification

$$
\mathscr{F} \cong \mathscr{F}_{V} \otimes \mathscr{F}_{\sim V}
$$

induced in momentum space by identifying $\ell_{2}((2 \pi / V) Z)$ with the functions in $\mathscr{L}_{2}(R)$ that are piecewise constant on intervals of length $2 \pi / V$ centered about the points in $(2 \pi / V) Z \equiv Z_{V}$. Thus we may regard $\varphi_{V}$ and $\mathfrak{A}(\mathscr{B}, V)$ as acting on $\mathscr{F}$.

For any volume $V$, with $\mathscr{B} \subset[-V / 2, V / 2]$, there is an isomorphism $\varrho_{V}$ of $\mathfrak{U}_{0}(\mathscr{B})$ onto $\mathfrak{A}_{0}(\mathscr{B}, V)$ generated by the correspondence

$$
\varphi(f) \rightarrow \varphi_{V}(f) ; \quad \pi(f) \rightarrow \pi_{V}(f) .
$$

We have not determined whether $\varrho_{V}$ is ultraweakly continuous, but a natural proof awaits a boson version of the Powers-Størmer result [6]. See [8-9].

Lemma 1.1. Let $A \in \mathfrak{U}_{0}(\mathscr{B})$. Then $\left\|\varrho_{V}(A)\right\|=\|A\|$ and as $V \rightarrow \infty$, st. $\lim \varrho_{V}(A)=A$.

Proof. Isomorphisms between $C^{*}$-algebras preserve norm; hence $\left\|\varrho_{V}(A)\right\|=\|A\|$. To prove the second statement, we may take $A$ a generator of $\mathfrak{A}_{0}(\mathscr{B})$, as in (1.2). By the semigroup convergence theorem, it is sufficient to prove convergence of the fields, $\varphi_{V}(f) \rightarrow \varphi(f)$ and $\pi_{V}(f) \rightarrow \pi(f)$, strongly on a core for $\varphi(f)$ and $\pi(f)$. However this convergence is well known, and can be shown by standard calculations, as in [5].

\section{The Locally Fock Property}

Each state $\omega$ on the $C^{*}$-algebra $\mathfrak{A}$ gives rise to a ${ }^{*}$-representation $\varrho_{\omega}$ of $\mathfrak{U}$ by bounded operators on a Hilbert space $\mathscr{H}_{\omega}$ (the GNS construction). We say $\omega$ is locally Fock if for each bounded open interval $\mathscr{B}$, the restriction $\omega \uparrow \mathfrak{H}(\mathscr{B})$ is normal (ultraweakly continuous). In this case $\varrho_{\omega} \uparrow \mathfrak{A}(\mathscr{B})$ is unitarily implemented; there is a unitary transformation $U_{\mathscr{B}}$ of Fock space into $\mathscr{H}_{\omega}$ such that

$$
\varrho_{\omega}(A)=U_{\mathscr{B}} A U_{\mathscr{B}}^{*}, \quad A \in \mathfrak{A}(\mathscr{B}) .
$$


This follows from the fact that $\mathfrak{U}(\mathscr{B})$ is a type III factor, and alternately it follows from the more easily verified fact that each $\mathfrak{A}(\mathscr{B})$ contains a type $\mathrm{I}_{\infty}$ factor. We note that a normal state on $\mathfrak{U}_{0}(\mathscr{B})$ has a unique extension to a normal state on its weak closure, $\mathfrak{A}(\mathscr{B})$, and so a locally Fock state on $\mathfrak{U}_{0}$ yields a locally Fock state on $\mathfrak{A}$.

The Hamiltonian $H_{V}$ has an eigenvector $\Omega_{V}$ satisfying $H_{V} \Omega_{V}=0$, $\left\|\Omega_{V}\right\|=1$. Since $\Omega_{V}$ is unique up to a phase, $\Omega_{V}$ is an eigenvector for the momentum operator $P_{V}$ for the volume $V$. Reasoning by continuity in the coupling constant and using the fact that $P_{V}$ has pure discrete spectrum, we see that the eigenvalue is zero, so $P_{V} \Omega_{V}=0$. We define the state $\omega_{V}$ on the algebra $\mathfrak{B}(\mathscr{F})$ of all bounded operators on $\mathscr{F}$ by

$$
\omega_{V}(A)=\left\langle\Omega_{V}, A \Omega_{V}\right\rangle .
$$

This state is invariant under the periodic space translation group with generator $P_{V}$. Likewise we define the state $\tilde{\omega}_{V}=\omega_{V} \varrho_{V}$ on $\mathfrak{A}_{0}(\mathscr{B})$. As $V \rightarrow \infty$, the states $\tilde{\omega}_{V}$ are defined eventually on a dense subalgebra of $\mathfrak{H}_{0}$. Thus by standard compactness arguments, there is a $w^{*}$-convergent subnet $\left\{\tilde{\omega}_{V_{v}}\right\}$, tending to a state $\omega$ defined on all of $\mathfrak{A}_{0}$. Fock.

Theorem 2.1. Any $w^{*}$-limit point $\omega$ of the sequence $\left\{\tilde{\omega}_{V}\right\}$ is locally

Our proof is based on the methods of [3], and we begin by outlining those ideas. In [3] we established the locally Fock property for a vacuum state defined through the limit $g \rightarrow 1$. The Hamiltonian $H(g)$ of $(1.3)$ has a ground state eigenvector $\Omega_{g}, H(g) \Omega_{g}=0,\left\|\Omega_{g}\right\|=1$. We let $g_{n}(x)=g(x / n)$, hold $g$ fixed and let $n \rightarrow \infty$. The approximate vacuum state $\omega_{n}$ is defined on $\mathfrak{A}$ as the average over spatial translations (by a distance up to $0(n)$ ) of the states $A \rightarrow\left\langle\Omega_{g_{n}}, A \Omega_{g_{n}}\right\rangle$. The spatial average ensures translation invariance of a limit point $\omega$ of the sequence $\left\{\omega_{n} \uparrow \mathfrak{A}\right\}$. A norm limit of normal states is normal, and $\omega_{n} \uparrow \mathfrak{A}(\mathscr{B})$ is normal, so the essential step in proving the locally Fock property is to show that the sequence $\left\{\omega_{n} \uparrow \mathfrak{A}(\mathscr{B})\right\}$ lies in a norm compact set of states.

Let $\mathscr{C}$ be a bounded interval of length $|\mathscr{C}| \geqq 1$. For $0 \leqq \tau<\frac{1}{4}$, we define the local number operator

where

$$
N_{\tau, \mathscr{C}}=\int a^{*}(p) w_{\tau, \mathscr{C}}(p, q) a(q) d p d q
$$

$$
w_{\tau, \mathscr{C}}(p, q)=(2 \pi)^{-1} \int E_{\mathscr{C}}(p-l) \mu(l)^{\tau} E_{\mathscr{C}}(l-q) d l
$$

and $E_{\mathscr{C}}$ is the Fourier transform of the characteristic function of $\mathscr{C}$. Note that $N_{0, \mathscr{C}}$ measures the number of particles in $\mathscr{C}$ and that $N_{0, \mathscr{C}} \leqq$ const. $N_{\tau, \mathscr{C}}$.

The locally Fock and norm compact properties are a consequence of the estimate

$$
\omega_{n}\left(N_{\tau, \mathscr{C}}\right) \leqq \text { const. }|\mathscr{C}| .
$$


We use (2.4) to define a truncated vacuum state $\omega_{n}^{\varepsilon}$ on $\mathfrak{B}(\mathscr{F})$,

$$
\omega_{n}^{\varepsilon}(A)=\omega_{n}(R A R),
$$

where $R$ is the projection onto vectors in $\mathscr{F}$ with at most $M\left(1+j^{2}\right)$ particles localized in the space interval $\mathscr{X}_{j}=[j, j+1], j=0, \pm 1, \ldots$. Hence $R$ projects onto vectors with at most $0\left(d^{3}\right)$ particles at a distance $d$ from the origin. For $M=M(\varepsilon)$ sufficiently large, the estimate

$$
\left\|\omega_{n}^{\varepsilon}-\omega_{n}\right\| \leqq \varepsilon
$$

follows from (2.4), see [3].

There is no uniform bound on the number of particles in the wave functions defining $\omega_{n}^{\varepsilon}$, and in order to achieve this result, we introduce a new approximation, $\omega_{n}^{\varepsilon \delta}$, all of whose wave functions are supported in a sufficiently large interval $\mathscr{C}=\mathscr{C}(\mathscr{B}, \delta, \varepsilon)$ containing $\mathscr{B}$. We take $\mathscr{C}$ to be a union of the $\mathscr{X}_{j}^{\prime}$ s. In the approximation $\omega_{n}^{\varepsilon \delta}$ of $\omega_{n}^{\varepsilon}$, the error $\omega_{n}^{\varepsilon}-\omega_{n}^{\varepsilon \delta}$ is small only on the local subalgebra $\mathfrak{A}(\mathscr{B})$ :

$$
\begin{aligned}
& \left\|\left(\omega_{n}^{\varepsilon \delta}-\omega_{n}^{\varepsilon}\right) \uparrow \mathfrak{U}(\mathscr{B})\right\|<\delta, \\
& \left\|\left(\omega_{n}^{\varepsilon \delta}-\omega_{n}\right) \uparrow \mathfrak{A}(\mathscr{B})\right\|<\varepsilon+\delta .
\end{aligned}
$$

To define $\omega_{n}^{\varepsilon \delta}$, we note that $\omega_{n}^{\varepsilon}$ is a normal state, and hence a sum of vector states. Let $\langle\theta, \cdot \theta\rangle$ be a vector state contributing to $\omega_{n}^{\varepsilon}$ and let $\theta_{i j}$ be the component of $\theta$ with $i$ particles in $\mathscr{C}, j$ particles in $\sim \mathscr{C}$. In configuration space variables, $\theta_{i j}=\theta_{i j}\left(x_{1} \ldots, x_{i}, y_{1}, \ldots, y_{j}\right), x_{\alpha} \in \mathscr{C}, y_{\beta} \in \sim \mathscr{C}$. We now regard $\theta_{i j}$ as an $i$ particle state in $\mathscr{F}$, depending on parameters $y_{1}, \ldots, y_{j}$. We thereby eliminate particles outside of $\mathscr{C}$ from $\theta$ and from $\omega_{n}^{\varepsilon}$. The contribution of $\langle\theta, A \theta\rangle$ to $\omega_{n}^{\varepsilon \delta}(A)$ is

$$
\sum_{j, i_{1} i_{2}} \int\left\langle\theta_{i_{1} j}, A \theta_{i_{2} j}\right\rangle d y_{1} \ldots d y_{j} .
$$

where $A$ acts on $\theta_{i_{1} j}$ and $\theta_{i_{2} j}$ as $i_{1}$ and $i_{2}$ particle states. We achieve an equivalent definition of $\omega_{n}^{\varepsilon \delta}$ by eliminating from $A$ its action on particles outside $\mathscr{C}$. We have $\mathscr{F}^{\mathscr{C}} \subset \mathscr{F}, \mathscr{F}^{\sim \mathscr{C}} \subset \mathscr{F}$ and

$$
\mathscr{F} \cong \mathscr{F}^{\mathscr{C}} \otimes \mathscr{F}^{\sim \mathscr{C}}
$$

where $\mathscr{F}^{\mathscr{C}}$ is the Fock space over $\mathscr{L}_{2}(\mathscr{C})$. We use a superscript $\mathscr{C}$ to denote the natural imbedding

$$
\mathscr{L}_{2}(\mathscr{C}) \subset \mathscr{L}_{2}\left(R^{1}\right), \quad \mathscr{F}^{\mathscr{C}} \subset \mathscr{F},
$$

obtained by extending $f \in \mathscr{L}_{2}(\mathscr{C})$ to a function identically zero on $\sim \mathscr{C}$. (This is distinct from the periodic imbedding $\mathscr{F}_{V} \subset \mathscr{F}$.) Let $Q^{\mathscr{E}}$ be the projection of $\mathscr{F}$ onto $\mathscr{F}^{\mathscr{C}}$. Then $Q^{\mathscr{C}} A Q^{\mathscr{C}}$ acts on $\mathscr{F}^{\mathscr{C}}$, and $Q^{\mathscr{C}} A Q^{\mathscr{C}} \otimes I$ acts on $\mathscr{F}^{\mathscr{C}} \otimes \mathscr{F}^{\sim \mathscr{C}}$. If $A^{\delta}$ is the corresponding operator on $\mathscr{F}$, under the 
isomorphism (2.8), then

$$
\omega_{n}^{\varepsilon \delta}(A)=\omega_{n}^{\varepsilon}\left(A^{\delta}\right)=\omega_{n}^{\varepsilon}\left(Q^{\mathscr{C}} A Q^{\mathscr{C}} \otimes I\right) .
$$

In other words, $\omega_{n}^{\varepsilon \delta}$ is the state which results from first projecting $\omega_{n}^{\varepsilon}$ onto a state on $\mathfrak{B}\left(\mathscr{F}^{\mathscr{C}}\right)$ by an integration over variables localized outside $\mathscr{C}$ and then tensoring the result with the no particle (bare vacuum) state on $\mathfrak{B}\left(\mathscr{F}^{\sim \mathscr{C}}\right)$.

The algebra $\mathfrak{A}(\mathscr{B})$, however, is not contained in $\mathfrak{B}\left(\mathscr{F}^{\mathscr{C}}\right)$ and the proof of (2.6) involves an estimate on the difference between the two notions of localization. The algebra $\mathfrak{B}\left(\mathscr{F}^{\mathscr{C}}\right)$ is localized with respect to the support of the configuration space wave functions in $\mathscr{F}$, while the algebra $\mathfrak{A}(\mathscr{B})$ is defined by localization of the time zero fields $\pi(x)$ and $\varphi(x)$. Due to the energy factors $\mu(p)^{ \pm \frac{1}{2}}$ which occur in the definition of $\pi(x)$ and $\varphi(x)$, the configuration space localization of the fields $\pi$ or $\varphi$ involves convolution of the wave functions by one of the kernels

$$
k^{ \pm}(x)=(2 \pi)^{-1} \int \mu(p)^{ \pm \frac{1}{2}} e^{-i p x} d p .
$$

The kernels $k^{ \pm}(x)$ are $\mathfrak{C}^{\infty}$ except at $x=0$, and outside of a neighborhood of $x=0$, they satisfy

$$
\left|k^{ \pm}(x)\right| \leqq 0(1) e^{-m|x|}
$$

Hence the difference between the localization of $\varphi(x)$ and $\pi(x)$ and the localization of $\mathfrak{B}\left(\mathscr{F}^{\mathscr{C}}\right)$ involves an exponentially small tail. A lengthy analysis of this effect yields (2.6), see [3].

Finally we prove that the sequence $\omega_{n}^{\varepsilon \delta}$ lies in a norm compact set of states. We use (2.4) with $\mathscr{C}$ replaced by $\mathscr{X}_{j}$ and the facts that $N_{\tau, \mathscr{X}_{j}}$ commutes with $R$ and that bounded functions of $N_{\tau, \mathscr{x}_{J}}$ belong to $\mathfrak{B}\left(\mathscr{F}^{\mathscr{C}}\right)$ to show that

$$
\sum_{\mathscr{X}_{j} \subset \mathscr{C}} \omega_{n}^{\varepsilon \delta}\left(N_{\tau, x_{j}}\right) \leqq \text { const. }|\mathscr{C}| .
$$

This estimate and the fact that

$$
\sum_{x_{j} \subset \mathscr{C}} N_{\tau, x_{j}}
$$

has a compact resolvent on $\mathscr{F}^{\mathscr{C}}$ is sufficient to establish norm compactness of the states $\omega_{n}^{\varepsilon \delta}$, as states on $\mathfrak{B}(\mathscr{F})$. The norm compactness of the sequence $\left\{\omega_{n} \backslash \mathfrak{A}(\mathscr{B})\right\}$ then follows.

We now use this construction for the periodic field $\varphi_{V}$ and the approximate vacuum state $\omega_{V}$ of (2.1). There are three steps in the argument: the approximations $\omega_{V} \rightarrow \omega_{V}^{\varepsilon}$ and $\omega_{V}^{\varepsilon} \rightarrow \omega_{V}^{\varepsilon \delta}$ and the existence of a norm compact set of states on $\mathfrak{B}(\mathscr{F})$ containing the family of states $\left\{\omega_{V}^{\varepsilon \delta}: V \geqq\right.$ const. $\}$. The vacuum $\Omega_{V}$ belongs to $\mathscr{F}_{V}[2]$ and the first two steps, with $V$ held fixed, take place entirely within $\mathscr{F}_{V}$. In the third step, 
$V \rightarrow \infty$, and we must work on the full Fock space $\mathscr{F}$. For an interval $\mathscr{C} \subset[-V / 2, V / 2]$ we have the Fock space $\mathscr{F}_{V}^{\mathscr{C}}$ of elements of $\mathscr{F}_{V}$ which are localized in $\mathscr{C}$. This notion of localization is defined by restricting the momentum space wave functions to the lattice $Z_{V}$ and then inverting the Fourier series to obtain periodic configuration space wave functions with support in $\mathscr{C}$. In terms of the identification $\mathscr{F}_{V} \subset \mathscr{F}$, this notion of localization is $V$ dependent. Elements of $\mathscr{F}_{V}^{\mathscr{E}}$ and $\mathscr{F}_{V}$ yields functions in $\mathscr{F}$ with piecewise constant Fourier transforms. Such functions never have compact support in the sense of $\mathscr{F}$ localization and cannot belong to $\mathscr{F}^{\mathscr{C}}$. We replace (2.8) with the representation $\mathscr{F}_{V} \cong \mathscr{F}_{V}^{\mathscr{C}} \otimes \mathscr{F}_{V}^{\sim \mathscr{C}}$, when working with a fixed $V$.

For $0 \leqq \tau<\frac{1}{4}$ and $\mathscr{C} \subset[-V / 2, V / 2]$, we define

where

$$
\begin{aligned}
N_{\tau, V, \mathscr{C}} & =(2 \pi / V) \sum_{p, q \in Z_{V}} a_{V}(p)^{*} w_{\tau, V, \mathscr{C}}(p, q) a_{V}(q) \\
& =\int a(p)^{*} w_{\tau, V, \mathscr{C}}(p, q) a(q) d p d q
\end{aligned}
$$

$$
w_{\tau, V, \mathscr{C}}(p, q)=(2 \pi)^{-1} \int d l E_{\mathscr{C}}\left(p_{V}-l_{V}\right) \mu\left(l_{V}\right)^{\tau} E_{\mathscr{C}}\left(l_{V}-q_{V}\right),
$$

where $p_{V}$ is the lattice point nearest $p$ and where

$$
a_{V}(q)=(V / 2 \pi)^{\frac{1}{2}} \int_{-\pi / V}^{\pi / V} a(q+r) d r .
$$

The estimates of [3, Section 3] show that $N_{\tau, V, \mathscr{C}}$ is an operator, and as in [3], we have the estimate

$$
\omega_{V}\left(N_{\tau, V, \mathscr{C}}\right) \leqq \text { const. }|\mathscr{C}|,
$$

which replaces (2.4).

For convenience, we suppose that $V / 2$ is an integer, so that $[-V / 2$, $V / 2]$ is a union of $\mathscr{X}_{j}$ 's. Let $R_{V}$ be the projection onto vectors in $\mathscr{F}_{V}$ which have at most $M\left(1+j^{2}\right)$ particles localized in the interval $\mathscr{X}_{j}, \mathscr{X}_{j} \subset[-V / 2$, $V / 2]$. For $M=M(\varepsilon)$ sufficiently large (but independent of $V$ ),

$$
\omega_{V}\left(I-R_{V}\right) \leqq \frac{1}{3} \varepsilon^{2}
$$

as a consequence of (2.13). We define

and then

$$
\omega_{V}^{\varepsilon}(A)=\omega_{V}\left(R_{V} A R_{V}\right),
$$

$$
\left\|\omega_{V}^{\varepsilon}-\omega_{V}\right\| \leqq \varepsilon .
$$

As before, we choose $\mathscr{C}=\mathscr{C}(\mathscr{B}, \delta, \varepsilon)$ sufficiently large, and we require that $V$ is large enough so that $\mathscr{C} \subset[-V / 2, V / 2]$. We repeat our construction above, to obtain the state $\omega_{V}^{\varepsilon \delta}$, by regarding variables $y \in \sim \mathscr{C}$ as parameters and integrating them, as in (2.7). Let $Q_{V}^{\mathscr{C}}$ be the projection 
of $\mathscr{F}_{V}$ onto $\mathscr{F}_{V}^{\mathscr{C}}$. Then

$$
\omega_{V}^{\varepsilon \delta}(A)=\omega_{V}^{\varepsilon}\left(Q_{V}^{\mathscr{C}} A Q_{V}^{\mathscr{C}} \otimes I\right)=\omega_{V}^{\varepsilon}\left(A^{\delta}\right) .
$$

Our basic estimates replacing (2.6) are

$$
\begin{aligned}
& \left\|\left(\omega_{V}^{\varepsilon}-\omega_{V}^{\varepsilon \delta}\right) \uparrow \mathfrak{A}(\mathscr{B}, V)\right\|<\delta, \\
& \left\|\left(\omega_{V}-\omega_{V}^{\varepsilon \delta}\right) \uparrow \mathfrak{A}(\mathscr{B}, V)\right\|<\varepsilon+\delta .
\end{aligned}
$$

In order to establish (2.15), we must compare the notion of localization in $\mathfrak{U}(\mathscr{B}, V)$ with the notion of localization in $\mathfrak{B}\left(Q_{V}^{\mathscr{C}} \mathscr{F}_{V}\right)$. This is analogous to the case $V=\infty, Q_{\infty}^{\mathscr{C}}=Q^{\mathscr{C}}$, that led to (2.6). The energy factors $\mu\left(p_{V}\right)^{ \pm \frac{1}{2}}$ occur in $\pi_{V}$ and $\varphi_{V}$. For example with

we have

$$
\begin{aligned}
k_{V}^{ \pm}(x) & =V^{-1} \sum_{k \in Z_{V}} \mu\left(p_{V}\right)^{ \pm \frac{1}{2}} e^{-i p_{V} x} \\
& =\sum_{n=-\infty}^{\infty} k^{ \pm}(x+n V)
\end{aligned}
$$

$$
\begin{aligned}
\varphi_{V}(x) & =(4 \pi)^{-\frac{1}{2}} \int\left\{a(p)^{*}+a(-p)\right\} e^{-i p_{V} x} \mu\left(p_{V}\right)^{-\frac{1}{2}} d p \\
& =\int_{-V / 2}^{V / 2} k_{V}^{-}(x-y) \hat{\varphi}_{V}(y) d y
\end{aligned}
$$

where the (Newton-Wigner) field $\hat{\varphi}_{V}(y)$ creates and annihilates a wave function, localized at $y$ in the sense of $\mathscr{F}_{V}$ localization. By (2.10) and (2.17),

$$
\left|k_{V}^{ \pm}(x)\right| \leqq \text { const. } e^{-m|x|}, \quad \gamma<|x|<V / 2,
$$

for any $\gamma>0$. Furthermore $k_{V}^{ \pm}(x)$ is $\widetilde{C}^{\infty}$ away from the origin. Using these facts, we follow the proof of (2.6) in order to establish (2.15).

Next we turn to the third step in the proof of Theorem 2.1, namely the limit of the states $\omega_{V}^{\varepsilon \delta}$ as $V \rightarrow \infty$.

Lemma 2.2. Let $\varepsilon$ and $\delta$ be fixed. The sequence $\left\{\omega_{V}^{\varepsilon \delta}: 1 \leqq V\right\}$ of states of $\mathfrak{B}(\mathscr{F})$ lies in a norm compact set. Any limit point $\omega^{\varepsilon \delta}$ is normal.

Proof. Since we consider the norm topology, it is sufficient to work with sequences. Given any subsequence of the $\omega_{V}^{\varepsilon \delta}$, we must draw from it a subsubsequence that is norm convergent as $V \rightarrow \infty$. Since $V$ is not fixed, we must work on the full Fock space $\mathscr{F}$.

Let $\tau<\frac{1}{4}$. We show that the resolvent of the operator $N_{\tau, V, \mathscr{C}}$ converges strongly as $V \rightarrow \infty$. It is sufficient to prove that on the one particle space the resolvent of $w_{\tau, V, \mathscr{C}}$ converges strongly to the resolvent of $w_{\tau, \mathscr{C}}$. These operators are defined by the kernels (2.12) and (2.3) respectively. From [3, Eq. (3.1.11)] we have the inequality

$$
\left\|w_{\tau, \mathscr{C}} \theta\right\| \leqq \text { const. }\left\|\mu^{2 \tau+\varepsilon} \theta\right\| .
$$


It follows that $w_{\tau, \mathscr{C}}$ (and likewise $w_{\tau, V, \mathscr{C}}$ ) is defined on the domain $\mathfrak{C}_{0}^{\infty}$, and we take $w_{\tau, \mathscr{C}}$ to be the Friedrichs extension of $w_{\tau, \mathscr{C}} \uparrow \mathfrak{C}_{0}^{\infty}$.

To compare our $\mathscr{F}_{V}$ periodic configuration space localization in $w_{\tau, V, \mathscr{C}}$ with the nonperiodic $\mathscr{F}$ configuration space localization, we introduce the isometry

$$
U_{V}=T_{V}^{-1} X_{V} T: L_{2}(R) \rightarrow L_{2}(R)
$$

where $T$ is the $\mathscr{L}_{2}(R)$ Fourier integral transform, $X_{V}$ is the orthogonal projection of $\mathscr{L}_{2}(R)$ onto functions piecewise constant on lattice intervals in momentum space, and $T_{V}^{-1}$ is the inverse Fourier series mapping onto $\mathscr{L}_{2}\left(-\frac{1}{2} V, \frac{1}{2} V\right)$, imbedded in $\mathscr{L}_{2}(R)$ as functions with support in $\left[-\frac{1}{2} V, \frac{1}{2} V\right]$.

Let $S$ be the closed operator $\mu^{\tau / 2} E_{\mathscr{C}}$. Since the Friedrichs extension of $w_{\tau, \mathscr{C}} \uparrow \mathfrak{C}_{0}^{\infty}$ is the only positive self adjoint extension of $w_{\tau, \mathscr{C}} \uparrow \mathfrak{C}_{0}^{\infty}$ with domain in $\mathscr{D}(S)$,

$$
w_{\tau, \mathscr{C}}=S^{*} S \text {. }
$$

Similarly, let $\Delta_{V}$ be the operator $d^{2} / d x^{2}$ with periodic boundary conditions on $\mathscr{L}_{2}\left(-\frac{1}{2} V, \frac{1}{2} V\right)$, and let

Then

$$
\begin{gathered}
\mu_{V}^{\tau / 2}=\left(-\Delta_{V}+m^{2}\right)^{\tau / 4}, \quad S_{V}=\mu_{V}^{\tau / 2} E_{\mathscr{C}}, \\
\hat{S}_{V}=U_{V}^{*} S_{V} U_{V} .
\end{gathered}
$$

$$
w_{\tau, V, \mathscr{C}}=\hat{S}_{V}^{*} \hat{S}_{V}=U_{V}^{*}\left(S_{V}^{*} S_{V}\right) U_{V} .
$$

We note that on $\mathfrak{C}_{0}^{\infty} \times \mathfrak{C}_{0}^{\infty}$, the difference $S_{V}^{*} S_{V}-S^{*} S$ is bounded and

$$
\left\|S_{V}^{*} S_{V}-S^{*} S\right\| \leqq 0\left(V^{-1}\right) \text {. }
$$

Hence by the resolvent equation, the resolvent $R_{V}(\zeta)=\left(S_{V}^{*} S_{V}-\zeta\right)^{-1}$ of $S_{V}^{*} S_{V}$ converges in norm to the resolvent $R$ of $S^{*} S=w_{\tau, \mathscr{C}}$. But $U_{V} \rightarrow I$ and $U_{V}^{*} \rightarrow I$ strongly, so

$$
\hat{R}_{V}(\zeta)=U_{V}^{*} R_{V}(\zeta) U_{V}=\left(w_{\tau, V, \mathscr{C}}-\zeta\right)^{-1}
$$

converges strongly as $V \rightarrow \infty$ to $R(\zeta)=\left(w_{\tau, \mathscr{C}}-\zeta\right)^{-1}$. Moreover, this proof shows that as $V \rightarrow \infty$, the resolvent of

$$
A_{V}=\sum_{x_{j} \subset \mathscr{C}} N_{\tau, V, x_{j}}
$$

converges strongly to the resolvent of

$$
A=\sum_{\mathscr{X}_{j} \subset \mathscr{C}} N_{\tau, \mathscr{X}_{j}} .
$$

Since $A \uparrow \mathscr{F}^{\mathscr{C}}$ has a compact resolvent [3] and since

$$
A \cong\left(A \uparrow \mathscr{F}^{\mathscr{C}}\right) \otimes I,
$$


$A$ has pure discrete spectrum with no accumulation points and with infinite multiplicity. The same conclusions apply to $A_{V}$. The usual contour integral formula shows that eigenspaces and eigenvalues converge, as $V \rightarrow \infty$.

We restrict the operators to the single particle space, to get more detailed information. On the single particle space the nonzero eigenvalues of $A_{V}$ or $A$ have finite multiplicity, and when the eigenvalues are numbered in increasing order, counting multiplicity, they converge as $V \rightarrow \infty$. Also the eigenvectors converge. We construct a basis for $\mathscr{F}_{\mathscr{C}}^{\mathscr{C}}$ and $\mathscr{F}_{V}^{\mathscr{C}}$ using tensor products of one particle eigenfunctions of $A\left\lceil\mathscr{F}^{\mathscr{C}}\right.$ and $A_{V} \uparrow \mathscr{F}_{V}^{\mathscr{C}}$. Since the single particle eigenfunctions converge as $V \rightarrow \infty$, so do their tensor products. These tensor products are eigenfunctions for $A$ or $A_{V}$. Thus we have constructed a basis $\left\{e_{1, V}, e_{2, V}, \ldots\right\}$ for $\mathscr{F}_{V}^{\mathscr{E}}$ with the properties

$$
\begin{aligned}
e_{j} & =\lim _{V \rightarrow \infty} e_{j V} \text { exists, } \\
\mu_{j} & =\lim _{V \rightarrow \infty} \mu_{j, V} \text { exists, } \\
A_{V} e_{j V} & =\mu_{j, V} e_{j V} \rightarrow A e_{j}=\mu_{j} e_{j}, \\
0 \leqq \mu_{1} & \leqq \mu_{2} \leqq \cdots \rightarrow \infty
\end{aligned}
$$

From the last statement, it follows that as $j \rightarrow \infty, \mu_{j, V} \rightarrow \infty$, uniformly in $V$.

The state $\omega_{V}^{\varepsilon \delta}$ is a normal state on $\mathfrak{B}(\mathscr{F})$, so there is a trace class matrix $\Lambda_{V}$ with trace norm $\left\|\Lambda_{V}\right\|_{1}=\left\|\omega_{V}^{\varepsilon \delta}\right\| \leqq 1$ and such that

$$
\omega_{V}^{\varepsilon \delta}(A)=\operatorname{Tr}_{\mathscr{F}}\left(\Lambda_{V} A\right), \quad A \in \mathfrak{B}(\mathscr{F}) .
$$

Now $\omega_{V}^{\varepsilon \delta}$ has the property

$$
\omega_{V}^{\varepsilon \delta}(A)=\omega_{V}^{\varepsilon \delta}\left(Q_{V}^{\mathscr{C}} A Q_{V}^{\mathscr{C}}\right),
$$

which implies that $\Lambda_{V}=Q_{V}^{\mathscr{C}} \Lambda_{V} Q_{V}^{\mathscr{C}}$. Thus $\Lambda_{V}^{\frac{1}{2}}$ can be expressed by a matrix $\left(\varrho_{i j}^{V}\right)$ in terms of the basis $\left\{e_{j, V}\right\}$. Hence by (2.13) and (2.20),

$$
\begin{aligned}
\omega_{V}^{\varepsilon \delta}\left(A_{V}\right)= & \sum_{i<j}\left(\mu_{i V}+\mu_{j V}\right)\left|\varrho_{i j}^{V}\right|^{2} \\
& +\sum_{i} \mu_{i V}\left|\varrho_{i i}^{V}\right|^{2} \\
\leqq & \text { const } .
\end{aligned}
$$

Passing to a subsequence, the $\varrho_{i j}^{V}$ 's converge as $V \rightarrow \infty$. Let

where

$$
\Lambda_{\vec{V}}^{\frac{1}{2}}=\varrho^{V, \kappa}+\delta \varrho^{V}
$$

$$
\varrho^{V, \kappa}=\sum_{i, j \leqq \kappa} \varrho_{i j}^{V} e_{i, V} \otimes e_{j, V}
$$


and similarly $\delta \varrho^{V}$ is the tail of the series. Then using the Hilbert Schmidt norm we have

$$
\left\|\Lambda_{\bar{V}}^{\frac{1}{2}}-\Lambda_{\bar{V}}^{\frac{1}{2}}\right\|_{2} \leqq\left\|\varrho^{V, \kappa}-\varrho^{V^{\prime}, \kappa}\right\|_{2}+\left\|\delta \varrho^{V, \kappa}\right\|_{2}+\left\|\delta \varrho^{V^{\prime}, \kappa}\right\|_{2} .
$$

Because of the bound (2.21),

$$
\left\|\delta \varrho^{V, \kappa}\right\|_{2}+\left\|\delta \varrho^{V^{\prime}, \kappa}\right\|_{2} \leqq \gamma / 2
$$

for all $V, V^{\prime}$, if $\kappa$ is chosen large enough so that $\mu_{\kappa, V}>$ const. $\gamma^{-1}$, for all $V$. We now hold $\kappa$ fixed. The right side of (2.22) is bounded by

$$
\begin{aligned}
\sum_{i, j \leqq \kappa}\left(\left|\varrho_{i j}^{V}-\varrho_{i j}^{V^{\prime}}\right|+\right.\text { const. } & \left\|e_{i, V}-e_{i, V^{\prime}}\right\| \\
& \left.+ \text { const. }\left\|e_{j, V}-e_{j, V^{\prime}}\right\|\right)+\gamma / 2 \leqq \gamma
\end{aligned}
$$

for $V, V^{\prime}$ sufficiently large, in our subsequence. As in [3, Lemma 4.7],

$$
\begin{aligned}
\left|\left(\omega_{V}^{\varepsilon \delta}-\omega_{V^{\prime}}^{\varepsilon \delta}\right)(A)\right| & =\left|\operatorname{Tr}_{\mathscr{F}}\left(\left(\Lambda_{V}-\Lambda_{V^{\prime}}\right) A\right)\right| \\
& \leqq\|A\|\left\|\Lambda_{V}-\Lambda_{V^{\prime}}\right\|_{1} \\
& \leqq \text { const. }\|A\|\left\|\Lambda_{\vec{V}}^{\frac{1}{2}}-\Lambda_{\vec{V}}^{\frac{1}{2}}\right\|_{2} \\
& \leqq o(1)\|A\|,
\end{aligned}
$$

for $V, V^{\prime}$ in the subsequence. This completes the proof of the lemma.

Let $\omega$ be a $w^{*}$-limit point of the sequence $\left\{\tilde{\omega}_{V}: 1 \leqq V\right\}$. Then there is a subnet $\tilde{\omega}_{V(\alpha)} \rightarrow \omega$. By the norm compactness, Lemma 2.2 , there is a subsubnet such that the $\omega_{V}^{\varepsilon \delta}$ have a norm limit $\omega^{\varepsilon \delta}$, for all $\varepsilon, \delta=1 / n$ :

$$
\omega^{\varepsilon \delta}=\operatorname{norm} \lim _{\beta} \omega_{V(\alpha(\beta))}^{\varepsilon \delta} .
$$

Since the states $\omega^{\varepsilon \delta}$ are normal, Theorem 2.1 follows from our next result.

Lemma 2.3. $\left\|\left(\omega-\omega^{\varepsilon \delta}\right) \uparrow \mathfrak{A}_{0}(\mathfrak{B})\right\| \leqq \varepsilon+\delta$.

Remark. Assuming the result of Lemma 2.3, we use Theorem 2.1 to extend $\omega$ to a locally Fock state of $\mathfrak{A}(\mathscr{B})$. By Kaplansky's density theorem, $\omega-\omega^{\varepsilon \delta}$ has the same norm, when restricted to $\mathfrak{U}_{0}(\mathscr{B})$ or to $\mathfrak{U}(\mathscr{B})$. Hence Lemma 2.3 yields

$$
\left\|\left(\omega-\omega^{\varepsilon \delta}\right) \uparrow \mathfrak{A}(\mathscr{B})\right\| \leqq \varepsilon+\delta .
$$

Proof. As states on $\mathfrak{A}_{0}(\mathscr{B})$,

$$
\begin{aligned}
\omega^{\varepsilon \delta}-\omega= & \left(\omega^{\varepsilon \delta}-\omega^{\varepsilon \delta} \circ \varrho_{V}\right)+\left(\omega^{\varepsilon \delta}-\omega_{V}^{\varepsilon \delta}\right) \circ \varrho_{V} \\
& +\left(\omega_{V}^{\varepsilon \delta}-\omega_{V}\right) \circ \varrho_{V}+\tilde{\omega}_{V}-\omega .
\end{aligned}
$$


Since $\omega^{\varepsilon \delta}$ is normal, Lemma 1.1 ensures that the first term is $w^{*}$ convergent to zero as $V \rightarrow \infty$. By hypothesis, the last term is $w^{*}$ convergent to zero. By (2.23), the second term is norm convergent to zero. Thus

$$
\left(\omega^{\varepsilon \delta}-\omega\right) \uparrow \mathfrak{A}_{0}(\mathscr{B})=w_{V \rightarrow \infty}^{*} \lim \left(\omega_{V}^{\varepsilon \delta}-\omega_{V}\right) \circ \varrho_{V} \uparrow \mathfrak{A}_{0}(\mathscr{B}) .
$$

Since $\varrho_{V}$ is an isomorphism, we have by (2.16),

$$
\begin{aligned}
\left\|\left(\omega_{V}^{\varepsilon \delta}-\omega_{V}\right) \circ \varrho_{V} \uparrow \mathfrak{A}_{0}(\mathscr{B})\right\| & =\left\|\left(\omega_{V}^{\varepsilon \delta}-\omega_{V}\right) \uparrow \mathfrak{A}_{0}(\mathscr{B}, V)\right\| \\
& \leqq \varepsilon+\delta .
\end{aligned}
$$

Since $w^{*}$-limits cannot increase norms, the lemma follows from (2.26).

Remark. $\omega$ is also a $w^{*}$-limit of a subsequence of the $\tilde{\omega}_{V}$ 's. It is sufficient to prove this for $\omega \uparrow \mathfrak{U}_{0}(\mathscr{B})$ and $\tilde{\omega}_{V} \uparrow \mathfrak{U}_{0}(\mathscr{B})$. We use (2.25) to write $\left(\tilde{\omega}_{V}-\omega\right) \uparrow \mathfrak{A}_{0}(\mathscr{B})$ as a sum of four terms. The terms $\omega^{\varepsilon \delta}-\omega$ and $\left(\omega_{V}^{\varepsilon \delta}-\omega_{V}\right) \circ \varrho_{V}$ converge to zero as $\varepsilon=\delta=1 / n \rightarrow 0$, uniformly in $\mathrm{V}$. We now hold $\varepsilon=\delta=1 / n$ fixed and let $V \rightarrow \infty$. The term $\omega^{\varepsilon \delta}-\omega^{\varepsilon \delta} \circ \varrho_{V}$ converges to zero in this limit. The final term is $\left(\omega^{\varepsilon \delta}-\omega_{V}^{\varepsilon \delta}\right) \circ \varrho_{V}$. Here $V=V(\alpha)$ belongs to the convergent net. Lemma 2.2 gives us norm compactness, and we can choose a subsequence of the subnet such that $\omega^{\varepsilon \delta}-\omega_{V}^{\varepsilon \delta} \rightarrow 0$. By the diagonal process we can choose one subsequence for all $\varepsilon=\delta$ $=1 / n$. Hence as $V \rightarrow \infty$ through this subsequence, $\left(\tilde{\omega}_{V}-\omega\right) \uparrow \mathfrak{A}_{0}(\mathscr{B})$ is $w^{*}$-convergent to zero.

\section{The Hamiltonian and Momentum Operators}

We show that the space-time translation automorphism $\sigma_{a}=\sigma_{\tau, \alpha}$ defined previously [2] through the limit $g \rightarrow 1$ of the Hamiltonians $H(g)$ agrees with the automorphism defined here through the limit $V \rightarrow \infty$ of the Hamiltonians $H_{V}$.

Theorem 3.1. Let $\sigma_{a, V}$ be the two parameter automorphism group generated by the operators $H_{V}$ and $P_{V}$. Then for $A \in \mathfrak{A}_{0}(\mathscr{B})$,

$$
\sigma_{a}(A)=s-\lim _{V \rightarrow \infty} \sigma_{a, V}\left(\varrho_{V}(A)\right) .
$$

We also show that the vacuum state $\omega$ of Section 2 is translation invariant,

$$
\omega \circ \sigma_{a}=\omega .
$$

If $U_{V}(a)$ is the unitary group generated by $H_{V}$ and $P_{V}$, and $\tilde{\omega}_{V_{j}} \rightarrow \omega$, we prove that

$$
\omega\left(C \sigma_{a}(A)\right)=\lim _{j \rightarrow \infty} \omega_{V_{j}}\left(\varrho_{V_{j}}(C) U_{V_{j}}(a) \varrho_{V_{j}}(A)\right) .
$$


These results permit us to transfer the spectral condition, previously established for $V<\infty$ [4] to the limit $V \rightarrow \infty$. Let $A \rightarrow A_{\text {ren }}$ denote the GNS representation defined by $\omega$, and let $A_{\text {ren }}$ act on $\mathscr{F}_{\text {ren }}=\mathscr{H}_{\omega}$.

Theorem 3.2. There exist commuting self adjoint operators $H$ and $P$ on $\mathscr{F}_{\text {ren }}$ that generate space time translations. Thus for $a=(\tau, \alpha) \in R^{2}$ and $A \in \mathfrak{A}$,

$$
\begin{aligned}
\sigma_{a}(A)_{\mathrm{ren}} & =U(a) A_{\mathrm{ren}} U(a)^{*}, \\
U(a) & =\exp (i \tau H-i \alpha P) .
\end{aligned}
$$

Furthermore $H \Omega=0=P \Omega$, and $0 \leqq H ; 0 \leqq H^{2}-P^{2}$.

Remark. The inequalities state that the spectrum lies in the forward cone. The second inequality is one of the improvements in the $\mathscr{P}(\varphi)_{2}$ theory permitted by this paper. Another improvement is that the bounds of [4] on vacuum expectation values of products of $\varphi_{t}$ and $\varphi_{x}$ can now be transferred to the limit $V \rightarrow \infty$, see Theorem 3.3. We define the (nonnegative) mass operator $M$ by $M=\left(H^{2}-P^{2}\right)^{\frac{1}{2}}$. An important open question is whether $M$ has an isolated, positive eigenvalue.

Proof of Theorem 3.1. To interpolate between the $g$ and $V$ cutoffs, we introduce

$$
H(g, V)=H_{0, V}+\int_{-V / 2}^{V / 2}: \mathscr{P}\left(\varphi_{V}(x)\right): g(x) d x-E_{g} .
$$

We have the strong limits

$$
\begin{aligned}
\exp (i \tau H(g)) & =\text { st. } \lim _{V \rightarrow \infty} \exp (i \tau H(g, V)), \\
\exp (i \alpha P) & =\text { st. } \lim _{V \rightarrow \infty} \exp \left(i \alpha P_{V}\right)
\end{aligned}
$$

where $P$ generates space translation on $\mathscr{F}$. To prove (3.4), we appeal to the semigroup convergence theorem, and use strong convergence of the generators on a core. $\mathfrak{C}^{\infty}\left(H_{0}\right)$ is a core for $H(g)$, by Rosen's higher order estimates [7], and $H(g, V) \rightarrow H(g)$ strongly on $\mathfrak{C}^{\infty}\left(H_{0}\right)$ by elementary estimates [5]. For (3.5), we also use the core $\mathfrak{C}^{\infty}\left(H_{0}\right)$.

Let $A \in \mathfrak{H}_{0}(\mathscr{B}), \mathscr{B}_{|\tau|+|\alpha|} \subset[-V / 2, V / 2]$ and let $g$ equal 1 on $\mathscr{B}_{|\tau|+|\alpha|}$. By Lemma 1.1 and (3.4-5),

$$
\begin{aligned}
\sigma_{a}(A)= & \lim _{V \rightarrow \infty} \exp (i \tau H(g, V)) \exp \left(-i \alpha P_{V}\right) \varrho_{V}(A) \\
& \times \exp \left(i \alpha P_{V}\right) \exp (-i \tau H(g, V)) .
\end{aligned}
$$

Thus to complete the proof of Theorem 3.1, we only need to show that $H_{V}$ and $H(g, V)$ define the same dynamics on $\mathfrak{A}\left(\mathscr{B}_{|\alpha|}, V\right)$ for times $|t| \leqq|\tau|$. We use the Trotter formula. In the free periodic box dynamics (i.e. with the interaction polynomial $\mathscr{P}$ replaced by zero), the commutator function 
is given by the formula

and

$$
\begin{aligned}
\Delta_{V}\left(x-x^{\prime}, t-t^{\prime}\right) & =i\left[\varphi_{0 V}(x, t), \varphi_{0 V}\left(x^{\prime}, t^{\prime}\right)\right] \\
& =\sum_{n=-\infty}^{\infty} \Delta\left(x-x^{\prime}+n V, t-t^{\prime}\right) .
\end{aligned}
$$

$$
\begin{aligned}
\varphi_{0 V}(x, t)= & \int_{|x-y|<V / 2} \Delta_{V}(x-y, t) \pi_{V}(y) d y \\
& +\int_{|x-y|<V / 2} \frac{\partial \Delta_{V}}{\partial t}(x-y, t) \varphi_{V}(y) d y .
\end{aligned}
$$

Since $\Delta(x, t)=\Delta_{\infty}(x, t)$ vanishes outside the light cone, namely for $|t|<|x|$, it follows that

$$
\Delta_{V}(x, t)=0 \text { for }|t|<|x|<V / 2 .
$$

Thus the free time translation $\sigma_{t, V}^{0}$ with generator $H_{0, V}$ defines an automorphism with unit propagation speed. For $A \in \mathfrak{A}\left(\mathscr{B}_{|\alpha|}, V\right)$ and $|t| \leqq|\tau|$,

$$
\sigma_{t, V}^{0}(A)=\exp \left(i t H_{0, V}\right) A \exp \left(-i t H_{0, V}\right) \in \mathfrak{U}\left(\mathscr{B}_{|t|+|\alpha|}, V\right) .
$$

Furthermore the interaction dynamics $\sigma_{t, V}^{\mathrm{I}}$ with generator $H_{\mathrm{I}}(g, V)$ leaves each $\mathfrak{H}\left(\mathscr{B}_{s}, V\right)$ invariant,

$$
\exp \left(i t H_{\mathrm{I}}(g, V)\right) \mathfrak{A}\left(\mathscr{B}_{s}, V\right) \exp \left(-i t H_{\mathrm{I}}(g, V)\right)=\mathfrak{A}\left(\mathscr{B}_{s}, V\right)
$$

and on $\mathfrak{A}\left(\mathscr{B}_{s}, V\right)$ with $|s|<\tau$, our restriction on $g$ makes $\sigma_{t, V}^{\mathrm{I}}$ independent of $g$ (see [5]). We use the essential self adjointness of $H(g, V)$ and the Trotter product formula to establish the $g$ independence and unit propagation speed of $\sigma_{t, V}$, see [5]. Taking $g \equiv 1$ on $\left[-\frac{1}{2} V, \frac{1}{2} V\right]$ and zero elsewhere yields $H(g, V)=H_{V}+$ const., which completes the proof of Theorem 3.1.

Proof of (3.1) and (3.2). First we note that (3.1) follows from the case $C=I$ of (3.2). In fact since $U_{V}(a) \Omega_{V}=\Omega_{V}$,

$$
\omega_{V_{j}}\left(U_{V_{j}}(a) \varrho_{V_{j}}(A)\right)=\omega_{V_{j}}\left(\varrho_{V_{j}}(A)\right) \rightarrow \omega(A)
$$

for $A \in \mathfrak{A}_{0}(\mathscr{B})$, which, combined with (3.2), yields (3.1). We now establish (3.2). We have

$$
\begin{aligned}
\omega\left(C \sigma_{a}(\mathcal{A})\right)-\omega_{V}\left(\varrho_{V}(C)\right. & \left.\sigma_{a, V}\left(\varrho_{V}(A)\right)\right) \\
= & \left(\omega-\omega^{\varepsilon \delta}\right)\left(C \sigma_{a}(A)\right) \\
& +\omega^{\varepsilon \delta}\left(C \sigma_{a}(A)-\varrho_{V}(C) \sigma_{a, V}\left(\varrho_{V}(A)\right)\right) \\
& +\left(\omega^{\varepsilon \delta}-\omega_{V}^{\varepsilon \delta}\right)\left(\varrho_{V}(C) \sigma_{a, V}\left(\varrho_{V}(A)\right)\right) \\
& +\left(\omega_{V}^{\varepsilon \delta}-\omega_{V}\right)\left(\varrho_{V}(C) \sigma_{a, V}\left(\varrho_{V}(A)\right)\right) .
\end{aligned}
$$


The first and last terms in (3.5) are dominated in magnitude by $(\varepsilon+\delta)\|C\|\|A\|$, by (2.16), (2.24), and (3.6). We now hold $\varepsilon$ and $\delta$ fixed. The third term tends to zero as $V \rightarrow \infty$ through a subsequence, by Lemma 2.2 and the choice of $\omega^{\varepsilon \delta}$, since

$$
\left\|\varrho_{V}(C) \sigma_{a, V}\left(\varrho_{V}(A)\right)\right\| \leqq\|C\|\|A\| .
$$

The second term tends to zero, since $\omega^{\varepsilon \delta}$ is a normal state on $\mathfrak{B}(\mathscr{F})$ and since the operators converge ultrastrongly to zero. This follows from Lemma 1.1, Theorem 3.1 and the fact that the operators are bounded in norm, uniformly in $V$. This proves (3.2).

Proof of Theorem 3.2. This is proved exactly as in $[3,5]$, where we construct $H, P$ and $\mathscr{F}_{\text {ren }}$ with $0 \leqq H$. From (3.2), we have

$$
\text { spectrum }\{H, P\} \subset \liminf \operatorname{spectrum}\left\{H_{V_{j}}, P_{V_{j}}\right\},
$$

and the right hand side was bounded in [4].

Theorem 3.3. Let $\varphi_{v}=\partial_{x} \varphi$ or $\partial_{t} \varphi$. There is a Schwartz space norm $|\cdot|_{n}$ on $\mathscr{S}\left(R^{2 n}\right)$ such that

$$
\mid\left.\int\left\langle\Omega, \varphi_{v_{1}, \text { ren }}\left(x_{1}, t_{1}\right) \ldots \varphi_{v_{n}} \text {, ren }\left(x_{n}, t_{n}\right) \Omega\right\rangle f(x, t) d x d t|\leqq| f\right|_{n},
$$

and the above vacuum expectation value is obtained as $V \rightarrow \infty$ through a subsequence from the vacuum expectation values of the box cutoff theories.

We consider first a finite volume approximation to the space time averaged fields $\varphi(f)=\int \varphi(x, t) f(x, t) d x d t$, where $f=\bar{f} \in \mathfrak{C}_{0}^{\infty}\left(R^{2}\right)$. This operator is self adjoint and essentially self adjoint on the domain $\mathbb{C}^{\infty}\left(H_{0}\right)$. The above integral presentation is valid in the sense of bilinear forms on a dense domain, see [2]. Let $\varphi(f)_{\text {ren }}$ denote the corresponding self adjoint operator on $\mathscr{F}_{\text {ren }}$. Similarly, let $\varphi_{V}(f)$ be the self adjoint operator determined by the formula

$$
\varphi_{V}(f)=\int e^{i H_{V} t} \varphi_{V}(x) e^{-i H_{V} t} f(x, t) d x d t .
$$

Lemma 3.4. With $\varphi(f), \varphi_{V}(f)$ as above,

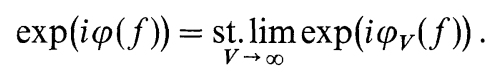

Proof. It is sufficient to prove convergence of $\varphi_{V}(f)$ on a core for $\varphi(f)$. Furthermore, as in the proof of Theorem 3.1, we may replace $H_{V}$ in (3.8) with $H(g, V)$, where $g \equiv 1$ on a sufficiently large interval. We choose the core $\mathfrak{C}^{\infty}(H(g))$ on which to estimate $\varphi_{V}(f)-\varphi(f)$.

In order to take advantage of the time integration, we note that for $H \equiv R^{-1} \equiv(H(g)+$ const. $)$,

$$
\varphi(f) \theta=R \varphi(f) H \theta+i R \varphi\left(f_{t}\right) \theta .
$$


Let $A=\int f(x, t) \varphi(x, 0) d x, B=i \int\left(\partial_{t} f\right)(x, t) \varphi(x, 0) d x$. Thus we calculate $(\delta \varphi) \theta=\left(\varphi_{V}(f)-\varphi(f)\right) \theta$ for $\theta \in \mathfrak{C}^{\infty}(H(g))$.

$$
\begin{aligned}
\delta(\varphi(f)) \theta= & \delta(R \varphi(f) H) \theta+\delta\left(i R \varphi\left(f_{t}\right)\right) \theta \\
= & \int \delta\left(e^{i t H}\right) R(A H+B) e^{-i H t} \theta d t \\
& +\int e^{i t H(V)}(\delta R)(A H+B) e^{-i t H} \theta d t \\
& +\int e^{i t H(V)} R_{V}\{(\delta A) H+\delta B\} e^{-i t H} \theta d t \\
& +\int e^{i t H(V)} R_{V} A_{V} \delta\left(e^{-i t H}\right) H \theta d t \\
& +\int e^{i t H(V)} R_{V} A_{V} e^{-i t H(V)}(\delta H) \theta d t \\
& +\int e^{i t H(V)} R_{V} B_{V} \delta\left(e^{-i t H}\right) \theta d t .
\end{aligned}
$$

Here $H(V)=H(g, V)+$ const. $=R_{V}^{-1}$,

$$
\delta A=A_{V}-A, \quad \delta B=B_{V}-B,
$$

etc. The first term converges to zero as $V \rightarrow \infty$ since $\delta\left(e^{i t H}\right)=e^{i t H(V)}-e^{i t H}$,

$$
\text { st. } \lim \delta\left(e^{i t \boldsymbol{H}}\right)=0,
$$

uniformly on compact $t$ intervals, and for $V_{i} \leqq \infty$,

$$
\left\|R_{V_{1}}^{\frac{1}{2}} A_{V_{2}}\right\|=\left\|R_{\vec{V}_{1}}^{\frac{1}{2}} B_{V_{2}}\right\| \leqq|f(\cdot, t)|+\left|\partial_{t} f(\cdot, t)\right|
$$

where $|f(\cdot, t)|$ denotes a suitable Schwartz space norm independent of $V_{i}$. By higher order estimates, $\|\delta R\| \rightarrow 0$ as $V \rightarrow \infty$ [7], so the second term converges to zero. Elementary $N_{\tau}$ estimates [5] yield

$$
\left\|(\delta A) R^{\frac{1}{2}}\right\|+\left\|(\delta B) R^{\frac{1}{2}}\right\| \leqq 0\left(V^{-1}\right)|f(\cdot t)| .
$$

Convergence of third term follows. The fourth and sixth terms converge on account of (3.9-10). We use the higher order estimate

$$
\left\|N^{r} R^{r}\right\| \leqq M_{r}, \quad r=1,2, \ldots
$$

to prove for $r \geqq \frac{1}{2} \operatorname{deg} P$,

$$
\begin{aligned}
\|\delta H \theta\| \leqq & \left\|\delta H_{\mathrm{I}} \theta\right\|+\left\|\delta H_{0} \theta\right\| \leqq\left\|\left(\delta H_{\mathrm{I}}\right)(N+I)^{-r}(N+I)^{r} \theta\right\| \\
& +\left\|\left(\delta H_{0}\right)(N+I)^{-1}(N+I) \theta\right\| \\
\leqq & \text { const. }\|\delta w\|_{2}\left\|H^{r} \theta\right\|+0\left(V^{-1}\right) \\
\leqq & 0\left(V^{-1}\right),
\end{aligned}
$$

and to establish convergence of the fifth term. Here $\delta w$ is the kernel $w_{V}-w$ of $\delta H_{\mathrm{I}}$. This completes the proof of the lemma. 
Let $h_{r} \in \mathfrak{C}_{0}^{\infty}\left(R^{2}\right), 1 \leqq r \leqq m$, let $C \in \mathfrak{A}_{0}(\mathscr{B})$ and let

$$
\begin{gathered}
A_{V}=\varrho_{V}(C) \prod_{r=1}^{m} \exp \left(i \varphi_{V}\left(h_{r}\right)\right) \\
A=C \prod_{i=1}^{m} \exp \left(i \varphi\left(h_{r}\right)\right) .
\end{gathered}
$$

Lemma 3.5. Let $\tilde{\omega}_{V_{j}} \rightarrow \omega$. Then

Proof.

$$
\omega(A)=\lim \omega_{V_{j}}\left(A_{V_{j}}\right) .
$$

$$
\begin{aligned}
\omega(A)-\omega_{V}\left(A_{V}\right)= & \left(\omega-\omega^{\varepsilon \delta}\right)(A)+\omega^{\varepsilon \delta}\left(A-A_{V}\right) \\
& +\left(\omega^{\varepsilon \delta}-\omega_{V}^{\varepsilon \delta}\right)\left(A_{V}\right)+\left(\omega_{V}^{\varepsilon \delta}-\omega_{V}\right)\left(A_{V}\right) .
\end{aligned}
$$

Note that $\|A\|=\left\|A_{V}\right\|=\|C\|$. We use (2.16) and (2.24) to bound the first and last terms, uniformly in $V$. We choose $\varepsilon$ and $\delta$ to be small, but fixed, and then the middle terms converge to zero by (2.23) and Lemma 3.4.

Proof of Theorem 3.3. We have an estimate similar to (3.7), uniform in $V$, on the vacuum expectation values in the box cutoff theory, $V<\infty$ [4]. Let $f_{i} \in \mathfrak{C}_{0}^{\infty}\left(R^{2}\right)$ and let

$$
\begin{aligned}
\theta & =\varphi_{v_{1}, \text { ren }}\left(f_{1}\right) \ldots \varphi_{v_{n}, \text { ren }}\left(f_{n}\right) \Omega, \\
\theta_{V} & =\varphi_{V, v_{1}}\left(f_{1}\right) \ldots \varphi_{V, v_{n}}\left(f_{n}\right) \Omega_{V} .
\end{aligned}
$$

By induction on $n$, assume that $\theta$ above is defined, $\|\theta\| \leqq\left|\mathrm{f}_{1} \otimes \cdots \otimes f_{n}\right|_{n}$, and for any choice of $C, m$ and $h_{r}$ in Lemma 3.5, assume that

$$
\left\langle\Omega, A_{\text {ren }} \theta\right\rangle=\lim _{j}\left\langle\Omega_{V_{j}}, A_{V_{j}} \theta_{V_{j}}\right\rangle .
$$

For $n=0$, take $\theta=\Omega, \theta_{V}=\Omega_{V}$ and then the induction hypothesis is valid by Lemma 3.5. To establish the hypothesis for $(n+1)$ consider

$$
F_{V}(s)=\left\langle\Omega_{V}, A_{V} \exp \left(i s \varphi_{v, V}(f)\right) \theta_{V}\right\rangle
$$

with $f \in \mathbb{C}_{0}^{\infty}$. By the induction hypothesis, $F_{V}(s)$ has a pointwise limit

$$
F(s)=\left\langle\Omega, A_{\text {ren }} \exp \left(i s \varphi_{v, \text { ren }}(f)\right) \theta\right\rangle .
$$

By the cutoff estimates [4], we have bounds

$$
\left|\left(\frac{d}{d s}\right)^{\alpha} F_{V}(s)\right| \leqq\|C\|\left|f \otimes f \otimes \cdots \otimes f \otimes f_{1} \otimes \cdots \otimes f_{n}\right|
$$

uniform in $V$, with a Schwartz space norm depending on $n$ and $\alpha$. It follows that the family $\left\{D^{\alpha} F_{V}\right\}$ of derivatives is equicontinuous as $V \rightarrow \infty$, and hence the derivatives $D^{\alpha} F_{V}$ converge uniformly and $F(s) \in \mathfrak{C}^{\infty}$. 
However, the convergence of $F_{V}^{\prime}(s)$ gives the induction hypothesis for $(n+1)$. Here we use the fact that vectors $\left\{A_{\text {ren }} \Omega\right\}$ are dense in $\mathscr{F}_{\text {ren }}$ as $C$ ranges over $\bigcup_{\mathscr{B}} \mathfrak{A}_{0}(\mathscr{B})$. Choosing $A=I, s=0$, we have convergence as $V_{j} \rightarrow \infty$ and the bound (3.7) for product wave functions in $\mathfrak{C}_{0}^{\infty}$. The general bound (3.7) follows by the nuclear theorem.

\section{Consequences of a Uniform Mass Gap}

The Hamiltonian $H_{V}$ is renormalized so that $0 \leqq H_{V}, H_{V} \Omega_{V}=0$. Zero is a simple eigenvalue for $H_{V}$ and $H_{V} \uparrow \mathscr{F}_{V}$ has a compact resolvent [2]. Since $H_{V} \uparrow\left(\mathscr{F}_{V}\right)^{\perp} \geqq m$, there is a gap between zero and the infimum $E_{1}$ of the spectrum of $H_{V} \uparrow\left\{\Omega_{V}\right\}^{\perp}$. In the limit $V \rightarrow \infty$, we expect $E_{1}$ to converge to the mass of the lightest particle in the theory, so $E_{1}$ is called the mass gap. If $E_{1}=E_{1}(V)$ is bounded uniformly away from zero as $V \rightarrow \infty$, we say the theory has a uniform mass gap.

Theorem 4.1. If the $\mathscr{P}(\varphi)_{2}$ theory has a uniform mass gap, then the vacuum $\Omega$ of Section 3 is unique and the vacuum expectation values of products of field operators are tempered distributions. Thus in this case all Wightman axioms are satisfied with the possible exception of unitary operators to implement Lorentz rotations.

Proof. Let $A \in \mathfrak{A}_{0}(\mathscr{B})$ and let $c_{V}=\left\langle\Omega_{V}, \varrho_{V}(A) \Omega_{V}\right\rangle$. Then $c_{V_{j}} \rightarrow c$ $=\left\langle\Omega, A_{\text {ren }} \Omega\right\rangle$ and so $\left(A_{\text {ren }}-c I\right) \Omega \perp \Omega$. Furthermore the set of vectors

$$
\left(A_{\text {ren }}-c I\right) \Omega,
$$

as $A$ and $\mathscr{B}$ vary, span $\{\Omega\}^{\perp}$. Thus we prove that $\Omega$ is the unique ground state for $H$ if we prove that (4.1) lies in the range of the spectral projection for the interval $\left[E_{1}, \infty\right)$. By (3.2),

$$
\begin{aligned}
& \left\langle\left(A_{\text {ren }}-c I\right) \Omega, e^{i t H}\left(A_{\text {ren }}-c I\right) \Omega\right\rangle \\
& \quad=\lim _{j}\left\langle\left(\varrho_{V_{j}}(A)-c_{V_{j}} I\right) \Omega_{V_{j}}, e^{i t H_{V_{j}}}\left(\varrho_{V_{j}}(A)-c_{V_{j}} I\right) \Omega_{V_{j}}\right\rangle .
\end{aligned}
$$

Considered as functions of $t$, we have pointwise convergence and a uniform bound in $\mathscr{L}_{\infty}$. Thus the functions converge as distributions in $t$ and consequently so do their Fourier transforms. On the right side, the Fourier transforms have support in $\left[E_{1}, \infty\right)$, since

$$
\left(\varrho_{V_{j}}(A)-c_{V_{j}} I\right) \Omega_{V_{j}} \perp \Omega_{V_{j}} .
$$

Thus the left side also has a Fourier transform with support in $\left[E_{1}, \infty\right)$, and we have proved that $\Omega$ is unique. Moreover

$$
\text { spectrum } H \uparrow\{\Omega\}^{\perp} \subset\left[E_{1}, \infty\right) \text {. }
$$


Next we bound the vacuum expectation values. Let $\xi \in \mathscr{S}\left(R^{1}\right)$ have a Fourier transform $\tilde{\xi}(E)=\int e^{-i t E} \xi(t) d t$ with suppt $\tilde{\xi} \subset\left(-\infty, E_{1} / 2\right)$ and $\tilde{\xi} \equiv 1$ on $\left(-E_{1} / 3, E_{1} / 3\right)$. For any $f \in \mathscr{S}\left(R^{2}\right)$, we write

$$
f=f_{1}+f_{2}=\xi * f+(f-\xi * f) .
$$

Since $\tilde{f}_{2}(p, E)$ vanishes for $|E|<E_{1} / 3$,

$$
f_{2}=(d / d t) h \text {. }
$$

We assert that

$$
\varphi(f) \Omega=\text { const. } \Omega-\pi(h) \Omega,
$$

where $\varphi(f)=\int \varphi(x, t) f(x, \mathrm{t}) d x d t$.

Since $\varphi\left(f_{2}\right)=-\varphi_{t}(h)=-\pi(h)$, we need only show that $\varphi\left(f_{1}\right) \Omega$ $=$ const. $\Omega$. However for a bounded function $\eta$,

$$
\begin{aligned}
\tilde{\eta}(H) \varphi\left(f_{1}\right) \Omega & =\int \eta(t) e^{-i t H} \varphi\left(f_{1}\right) \Omega d t \\
& =\varphi\left(\eta * f_{1}\right) \Omega \\
& =\varphi(\eta * \xi * f) \Omega=\varphi(\xi * f) \Omega \\
& =\varphi\left(f_{1}\right) \Omega
\end{aligned}
$$

if $\tilde{\eta} \equiv 1$ on suppt $\tilde{\xi}$, suppt $\tilde{\eta} \subset\left(-2 E_{1} / 3,2 E_{1} / 3\right)$. For such an $\eta$, the range of $\eta(H)$ is one dimensional and spanned by $\Omega$, by (4.2), proving (4.5). The constant in (4.5) can be evaluated as

$$
\text { const. }=\int f(x, t) d x d t\langle\Omega, \varphi(0,0) \Omega\rangle .
$$

The right side is finite, by estimates such as (2.13), and defines a continuous functional on $\mathscr{S}$. Furthermore the mapping $f \rightarrow h$ is continuous on $\mathscr{S}$, since $f_{2}$ vanishes in a neighborhood of the origin. Thus given any Schwartz norm $|\cdot|_{s_{1}}$ there is another norm $|\cdot|_{s_{2}}$ with

$$
|h|_{s_{1}} \leqq|f|_{s_{2}} .
$$

We use (4.5) to convert the $\varphi_{x}$ and $\varphi_{t}=\pi$ estimates of Theorem 3.3 into $\varphi$ estimates. Let $\zeta \in \mathfrak{C}_{0}^{\infty}$ be a nonnegative function with support in the square $|x| \leqq 1,|t| \leqq 1$ and such that the translates $\zeta_{j}(x, t)=\zeta(\{x, t\}-j)$ by $j \in Z_{2}$ form a partition of unity:

$$
\sum_{j \in Z_{2}} \zeta_{j}=1
$$

Then $f \rightarrow \zeta_{j} f$ is continuous on $\mathscr{S}$ and for any given norm $|\cdot|_{s_{1}}$ on $\mathscr{S}$ and any positive integer $N$, there is a norm $|\cdot|_{s_{2}}$ on $\mathscr{S}$ such that

$$
\left|\zeta_{j} f\right|_{s_{1}} \leqq(|j|+1)^{-N}|f|_{s_{2}}, \quad \text { all } j \in Z_{2}
$$

In other words, the series $\sum_{j} \zeta_{j} f$ converges in $\mathscr{S}$. 
In place of the vacuum $\Omega$ in (4.5), we now consider a vector of the form

$$
\varphi_{v_{1}}\left(h_{1}\right) \ldots \varphi_{v_{n}}\left(h_{n}\right) \Omega
$$

where each $\varphi_{v_{1}}$ is a $\varphi_{x}$ or a $\varphi_{t}$. By Theorem 3.3 the vector (4.9) is a continuous function of $h_{1} \ldots h_{n} \in \mathscr{S}\left(R^{2}\right) \times \cdots \times \mathscr{S}\left(R^{2}\right)$. Also the series

$$
\sum_{j_{1} \in Z_{2}} \varphi_{v_{1}}\left(\zeta_{j_{1}} h_{1}\right) \ldots \varphi_{v_{n}}\left(\zeta_{j_{n}} h_{n}\right) \Omega
$$

converges in $\mathscr{S}$. We assert that each term belongs to the domain of $\varphi\left(\zeta_{j} f\right)$, for any $f \in \mathscr{S}\left(R^{2}\right)$. In fact with our local unitary equivalence ensured by the locally Fock property, we return to Fock space and recall that for $f_{1}$ and $f_{2} \in \mathfrak{C}_{0}^{\infty}\left(R^{2}\right), \varphi\left(f_{1}\right), \varphi\left(f_{2}\right)$ and $\varphi\left(f_{1}+f_{2}\right)$ are essentially self adjoint on a common domain. It follows that

$$
\mathscr{D}\left(\varphi\left(f_{1}\right)\right) \cap \mathscr{D}\left(\varphi\left(f_{2}\right)\right) \subset \mathscr{D}\left(\varphi\left(f_{1}+f_{2}\right)\right) .
$$

The inclusion (4.11) is then valid on $\mathscr{F}_{\text {ren }}$ also, and we see that $\varphi\left(f_{1}+f_{2}\right)$ $=\left(\varphi\left(f_{1}\right)+\varphi\left(f_{2}\right)\right)^{-}$. We return to (4.10), and we begin with the fact that $\Omega \in \mathscr{D}\left(\varphi\left(\zeta_{j} f\right)\right)$. If the space component $j^{1}$ of $j$ is large enough, $\varphi\left(\zeta_{j} f\right)$ commutes with the operators $\varphi_{v_{i}}\left(\zeta_{j_{i}}, h_{i}\right)$. Consequently, these operators map the domain $\mathscr{D}\left(\varphi\left(\zeta_{j} f\right)\right)$ into itself, proving our assertion in this case.

If $j-j_{i}$ is time like, then $\varphi\left(\zeta_{j} f\right)$ need not commute, but $\varphi\left(\zeta_{j} f\right)$ is the sum of a space translate that commutes and a gradient. In fact for $h_{\alpha}(x)=h(x-\alpha)$,

$$
\varphi(h)=\varphi\left(h_{\alpha}\right)+\int_{0}^{\alpha}(\nabla \varphi)\left(h_{\alpha^{\prime}}\right) d \alpha^{\prime} .
$$

A term in (4.10) belongs to the domain of the gradient $\varphi_{x}(h)$ by Theorem 3.3, and it belongs to the domain of the space translate of $\varphi\left(\zeta_{j}, f\right)$ by local commutativity, as above. By (4.11), the term belongs to $\mathscr{D}\left(\varphi\left(\zeta_{j} f\right)\right)$ and the assertion is proved in all cases. Combining this argument with (4.5), we have

$$
\begin{aligned}
\varphi\left(\zeta_{j} f\right) \varphi_{v_{1}}\left(\zeta_{j_{1}} h_{1}\right) \ldots \varphi_{v_{n}}\left(\zeta_{j_{n}} h_{n}\right) \Omega= & \text { const. } \varphi_{v_{1}}\left(\zeta_{j_{1}} h_{1}\right) \ldots \varphi_{v_{n}}\left(\zeta_{j_{n}} h_{n}\right) \Omega \\
& +\varphi_{v_{1}}\left(\zeta_{j_{1}} h_{1}\right) \ldots \varphi_{v_{n}}\left(\zeta_{j_{n}} h_{n}\right) \pi\left(g_{1}\right) \Omega \\
& +\varphi_{x}\left(g_{2}\right) \varphi_{v_{1}}\left(\zeta_{j_{1}} h_{1}\right) \ldots \varphi_{v_{n}}\left(\zeta_{j_{n}} h_{n}\right) \Omega .
\end{aligned}
$$

The constant is given by (4.6), independently of $j, j_{i}$ and $h_{i} . g_{1}$ and $g_{2}$ belong to $\mathscr{S}$, and for any norm $|\cdot|_{s_{1}}$ on $\mathscr{S}$, there is a norm $|\cdot|_{s_{2}}$ on $\mathscr{S}$ and an integer $M$ such that

$$
\left|g_{1}\right|_{s_{1}}+\left|g_{2}\right|_{s_{1}} \leqq\left(\sup _{i}\left|j-j_{i}\right|+1\right)^{M}|f|_{s_{2}}
$$

We can now sum the series (4.12) over $j$ and $\left(j_{1}, \ldots j_{n}\right) \in Z_{2} \times \cdots \times Z_{2}$. The series converges absolutely, and is dominated term by term by a 
series of the form

$\sum_{j, j_{i}}(|j|+1)^{-N}\left(\sup _{i}\left|j-j_{i}\right|+1\right)^{M}\left(\sup _{i}\left|j_{i}\right|+1\right)^{-N}\left|h_{1}\right|_{s_{2}} \ldots\left|h_{n}\right|_{s_{2}}|f|_{s_{2}}$

In this series, we first choose the norm $|\cdot|_{s_{1}}$ on $g_{1}$ and $g_{2}$ required by [4]. This norm determines an $M$. We then choose $|\cdot|_{s_{2}}$ by (4.13) and also by (4.8), so that $N>M+2 n$. In this case (4.14) converges, so (4.9) defines a vector in the domain of $\varphi\left(\zeta_{j} f\right)$. Furthermore, we define $\varphi(f)$ to be the closure of $\sum_{j} \varphi\left(\zeta_{j} f\right)$, and then (4.9) belongs to the domain of $\varphi(f)$ also.

By the nuclear theorem, we can replace the test function $h_{1} \otimes \cdots \otimes h_{n}$ $\in \mathscr{S}\left(R^{2}\right) \otimes \cdots \otimes \mathscr{S}\left(R^{2}\right)$ by a test function $h \in \mathscr{S}\left(R^{2 n}\right)$. We then have

$$
\begin{aligned}
\varphi(f)\left(\varphi_{v_{1}} \ldots \varphi_{v_{n}}\right)(h) \Omega= & \text { const. }\left(\varphi_{v_{1}} \ldots \varphi_{v_{n}}\right)(h) \Omega \\
& +\left(\varphi_{v_{1}} \ldots \varphi_{v_{n}} \pi\right)\left(h_{1}\right) \Omega \\
& +\left(\varphi_{x} \varphi_{v_{1}} \ldots \varphi_{v_{n}}\right)\left(h_{2}\right) \Omega .
\end{aligned}
$$

The constant is given by (4.6) and $h_{1}$ and $h_{2}$ depend continuously on $f$ and $h$ in the $\mathscr{S}$-topology. We use (4.15) to bound $\varphi\left(f_{1}\right) \ldots \varphi\left(f_{n}\right) \Omega$ by induction on $n$. This completes the proof.

We conclude with some comments on the status of the missing Wightman axioms. Bounds on the vacuum expectation values of products of fields $\varphi(x, t)$ would follow, for instance, from an estimate

$$
N_{\tau, V, \mathscr{C}} \leqq \text { const. }\left(H_{V}+I\right) \text {, }
$$

uniform in $V$, without consideration of the mass gap. It is possible that a Poincaré-invariant vacuum could be constructed by averaging over the Lorentz automorphism $\sigma_{A}$ of homogeneous Lorentz transformations of $\mathfrak{A}$. In the reverse direction, it is possible that a unique vacuum could be found by decomposing into components irreducible under $\mathfrak{A}$, see [1]. In either case, the problem would be to preserve the locally Fock property in the construction. Both constructions preserve the spectral condition. If the vacuum is unique, not just as a Hilbert space vector, but also as a $C^{*}$ algebra state on $\mathfrak{U}$, then it is Poincare invariant. If we are given a Poincaré invariant state $\omega$, the automorphisms $\sigma_{\{a, 1\}}$ are implemented by a continuous unitary representation $U(a, \Lambda)$ of the Poincare group.

\section{References}

1. Araki, H.: On the algebra of all local observables. Prog. Theor. Phys. 32, 844-854 (1964).

2. Glimm,J., Jaffe, A.: The $\lambda\left(\varphi^{4}\right)_{2}$ quantum field theory without cutoffs II: The field operators and the approximate vacuum. Ann. Math. 91, 362-401 (1970).

3. - - The $\lambda\left(\varphi^{4}\right)_{2}$ quantum field theory without cutoffs III: The physical vacuum. Acta Math. 125, 203-267 (1970). 
4. Glimm,J.,Jaffe, A.: The energy momentum spectrum and vacuum expectation values in quantum field theory. J. Math. Phys. 11, 3335-3338 (1970).

5. - - Field Theory Models, in the 1970 Les Houches Lectures, C. Dewitt and R. Stora, eds. New York: Gordon \& Breach Sci. Publ. 1971.

6. Powers, R., Størmer, E.: Free states of the canonical anticommutation relations. Commun. Math. Phys. 16, 1-33 (1970).

7. Rosen,L.: The $\left(\varphi^{2 n}\right)_{2}$ quantum field theory without cutoffs: Higher order estimates. Commun. Pure Appl. Math., to appear.

8. Van Daele,A.: Quasi-equivalence of quasi-free states on the Weyl algebra. Commun. Math. Phys. 21, 171-191 (1971).

9. Araki,H.: On quasifree states of the canonical commutation relations (II), to appear.

\title{
J. Glimm
}

Courant Institute of Mathematical Sciences

New York University

New York, N. Y. 10012, USA

\author{
A.Jaffe \\ Lyman Laboratory of Physics \\ Harvard University \\ Cambridge, Mass. 02138, USA
}

